\title{
CANCER SERVICES - THE PRIMARY HEALTHCARE TEAM PERSPECTIVE
}

\author{
Philip Buckler, General Practitioner, \\ James Cochrane Practice, Kendal
}

In this article I shall be considering the role played by primary care in the provision of services to patients with cancer. Much of this article would apply to cancer service provision anywhere in the UK but in the Morecambe Bay Health Authority there are certain aspects that are peculiar to the area, mainly local geography, spread of population and paucity of public transport to many of the rural areas.

It is estimated that Morecambe Bay Health Authority will expect to see approximately 1600 new cancer registrations each year. The majority of these will have presented initially to their general practitioner either with symptoms or following a positive result in a screening programme.

\section{THE PRIMARY HEALTHCARE TEAM - WHAT IS IT?}

The traditional family healthcare team comprises the GP, district nurse and health visitor. When considering the management of patients with cancer this list would have to be expanded to include the Macmillan nursing service, social services, charitable organisations and, more importantly, all those who will be caring for the patient especially in the terminal phase of the illness. These may include the patient's relatives, neighbours, carers employed by social services and staff working in both residential and nursing homes.

It is important to recognise that many of these carers will have had little or no previous experience of looking after patients with cancer and they will need support themselves. Another group sometimes overlooked is the reception staff at the GP's surgery. They can play a pivotal role as it can be very reassuring for patients and their carers to be able to ring the surgery and be dealt with sympathetically by the reception staff. This can be facilitated even further if they are able to ask for a nominated member of staff with whom they may build up a relationship, which can provide a useful communication link with the members of the primary healthcare team.

In the initial stages of the illness the GP will probably play a prominent role, but as the illness progresses others will play an increasingly important part so that by the terminal phase at home the district nurse will probably be the major healthcare team member.

\section{THE ROLE OF THE PRIMARY HEALTHCARE TEAM IN THE MANAGEMENT OF PATIENTS WITH CANCER}

The team is involved as follows:

1 health promotion and screening

\author{
2 diagnosis \\ 3 treatment \\ 4 terminal care \\ 5 post-bereavement counselling of relatives and friends
}

\section{Health promotion and screening}

The primary healthcare team is involved in health promotion and screening in a number of ways. Firstly, health promotion: GPs in the course of their routine consultation may be able to inquire about and give advice on such things as smoking, sun exposure and occupational hazards. They may know of familial problems and direct patients towards screening, eg those with a strong family history of breast and colonic cancer. Secondly, GP's are involved in the two national screening programmes currently running, ie cervical and breast screening. In cervical screening GPs have to encourage attendance for regular screening and chase up those who default. They also have to counsel and refer those who have a positive result. With regard to the screening mammography programme, the GP's initial involvement is largely administrative. All practices are sent lists of patients due to be screened and are asked to check these for inaccuracies, appropriateness of screening etc. This can be a very timeconsuming exercise if these lists are to be kept as accurate and up-to-date as possible. The mammography screening programme highlights one of the geographical problems in the Morecambe Bay Health Authority: the referral centre for patients who have a positive result is in Whitehaven. This can pose significant travelling difficulties for patients from south Cumbria and many ask for referral to their nearest district general hospital rather than have to travel to Whitehaven for further evaluation and treatment.

The largest group of patients by far who present for screening are the worried well. Often these patients will present after coverage of a particular type of cancer in the media, the most recent being the widespread publicity given to prostatic cancer and the role of the prostatic surface antigen (PSA) test as a possible screening tool. It is currently recognised that the PSA test is probably not an ideal screening test for prostatic cancer and explaining this to worried patients can be a very time-consuming business.

\section{Diagnosis of cancer}

Patients will present to their GP either with symptoms or as a result of participating in screening programme. The GP's role here is to refer the patient on to the appropriate consultant for further evaluation and treatment. In the Morecambe Bay Health Authority the great majority of these referrals will be made to one of the three district general hospitals rather than immediate referral to a specialist cancer hospital such as the Christie Hospital in Manchester. The possibility that a patient might have cancer will engender much anxiety in that patient, and the speed and ease with which he can see a consultant for further management is clearly very important. Once it is 
certain that the patient has cancer it is often appropriate to involve other agencies as well, to support not only the patient but also his relatives. In south Cumbria and north Lancashire many patients will be put in touch with CancerCare. This charitable organisation provides many services for the cancer patient and his relatives, including therapies to enhance the psychological welfare of the patient, support for relatives, and transport. If the patient has a cancer which may need the involvement of the district nursing service then it is often useful to involve the district nurses soon, so that they have the opportunity to get to know the patient and family and build up a rapport early in the patient's illness.

\section{Treatment}

The treatment that any patient requires clearly depends on which cancer he has and the degree to which it has spread. The majority of patients requiring surgical intervention and/or chemotherapy will be managed at their nearby district general hospital. For those patients requiring radiotherapy the majority are currently referred to the Christie hospital. This brings us to another of the problems that patients within the Morecambe Bay Health Authority will meet, which is the distance that some of them have to travel both for investigation and treatment. In my experience most of these patients express a wish to be referred to a centre of excellence despite the undoubted problems that some of them experience with transport. Certainly for my patients the centre of excellence for radiotherapy is perceived to be the Christie, and should the oncology unit at Preston get off the ground it will be interesting to see whether patients will be as happy to go there as they are now to go to the Christie.

The GP's role in the treatment of patients with cancer will be to explain to the patients what their cancer is, its likely prognosis and what the aims of treatment are. It goes without saying that in order to do this the GP must be fully informed by the hospital so that he can advise the patient accordingly. Often patients will have had very thorough explanations in the hospital but it is clear when they come to the GP or speak to a practice or district nurse that very little of this information has sunk in. Such counselling can be very timeconsuming. The treatment of cancer patients can be made more difficult if the patient himself denies the cancer, or if relatives have expressed a wish that under no circumstances should he be told of it. Most patients in this situation will in fact know that they do have cancer. Nowadays most health professionals feel that it is best that patients should know their diagnosis as this allows any discussion to be open and honest. This can be especially important during the terminal phase of the illness.

The primary healthcare team's involvement with the actual treatment will include investigative procedures, such as monitoring full blood counts on patients receiving chemotherapy, and the administration of drugs advised by the hospital unit caring for the patient. This can sometimes create problems, especially if a GP is asked to administer a drug with which he is unfamiliar. Protocols provided by the hospitals can be very useful in such situations.

\section{Terminal care}

A number of patients will not be cured by their treatment and as a consequence will probably enter a terminal illness related to their underlying cancer. Increasing numbers of patients are now writing advance directives and will have discussed and agreed these with their GP in advance. For terminal patients the main aim of treatment is to keep them as well as possible, both physically and emotionally. The treatments available to do this are discussed in another article in this journal. Many of the problems encountered by patients at this time can be handled adequately in the primary care/home setting. More and more patients now express a wish to be nursed at home during this phase of illness, particularly those in rural areas. Caring for these patients at home can be a heavy commitment. In my own practice we have seven fulltime equivalent district nurses and we estimate that at any time one and a quarter fulltime equivalents are devoted entirely to palliative care. Once the patient enters the terminal phase of his illness, then the nursing time required to look after him at home can increase significantly. This can place great strains on the district nursing service's resources and at times a difficult balance has to be struck between the needs of the terminally ill and those of other patients. Morecambe Bay Health Authority has two hospices within its territory, which provide a very useful specialist service for symptom control. In addition they will admit patients for short spells to give their carers some relief, and will admit a number of patients when they are dying. Many patients, however, are reluctant to go to a hospice and the reason cited will often be geography. It must be remembered that even in this day and age a significant proportion of the population does not own or have access to private transport. In this situation a patient's admission to a unit some miles away can create difficulties for relatives and friends wishing to visit. One might try to make a case for having a hospice in the vicinity of each of the three district general hospitals in our area: the drawback to this would be the fact that often the reason for a patient's referral to a hospice is because that hospice offers a particular area of expertise. In order to maintain the level of expertise, the hospice staff will need to see a certain number of patients on a regular basis. If their services are spread too thinly this would inevitably dilute their experience and expertise accordingly. Perhaps a way forward might be the development of "community based hospices" so that the hospice expertise can be based more locally and be more readily available to patients in their own homes. We must remember that the carers looking after patients in the terminal phase may be undertaking work that they do not feel properly equipped to do, and they will rely heavily on the district nurse and GP for advice and help. Relatives will often want to do far more than can reasonably be expected of them, and one of the roles of the primary healthcare team is to recognise this and address the problem. This may involve arranging a relief admission for the patient in order to give the carer a break, or it may mean involving other agencies, such as the night nursing service.

\section{After death}

In the period immediately after death, the relatives of the deceased patient will need support, which will normally be provided by the primary healthcare team. Often relatives will ask for more information about the illness, and in particular whether or not there are implications for future generations. Should the patient
$6 \mathbf{6}$ carers looking after patients in the terminal phase may be undertaking work that they do not feel properly equipped to do 
indeed have died from a cancer with a genetic component the GP will need to advise the family accordingly, and if there are relatives who live out of the area it would be appropriate for the GP to urge them to seek advice locally, and perhaps to write to their own GP if necessary. After bereavement a number of relatives benefit from ongoing involvement with organisations such as CancerCare. With more patients dying at home, increasingly the GP will be the one who completes the death certificate. It goes without saying that it is important that these are completed as accurately and fully as possible so that reliable mortality statistics can be collated both regionally and nationally.

\section{The future}

An increasing elderly population combined with a shift in healthcare provision from the hospital to the community, has resulted in the primary healthcare team becoming more involved with the care of patients at all levels. It would seem likely that in the future new screening programmes will be developed and new treatments become available. Genetic counselling may become more relevant to more people. All of this work could fall to the primary healthcare team, but to be done properly it will require commitment, training and more manpower, if the other areas of general practice are not to suffer.

Traditionally, charities have provided important financial contributions towards the purchase of specialist nursing equipment, research and support for cancer sufferers and their relations. With the advent of the national lottery their income has been significantly affected. This means that if the current level of service is to be maintained, let alone increased, there will need to be more provision from the health authorities. Time will tell ... 